\title{
Comparison of Reduction of Dental Plaque and Gingivitis between Herbal and Nonherbal Dentifrices and Mouth Rinses in Orthodontic Cases: A Randomized Single-blind Clinical Study
}

\author{
Bhagyalakshmi Avinash ${ }^{1}$, Avinash B Shivamallu², Nandlal Bhojraj ${ }^{3}$, Suma Shekar ${ }^{4}$
}

\begin{abstract}
Aim: The aim of this research was to examine the oral hygiene efficacy between herbal and nonherbal dentifrices and mouth wash in orthodontic cases.

Materials and methods: The study was a single center, single blind, parallel arm, randomized controlled clinical trial with an allocation ratio of 1:1. The sample size was 40 participants, irrespective of sex. For all the participants, oral hygiene status was assessed at three occasions during the study. The first assessment of plaque index (PI) and gingival index (GI) was done before starting the orthodontic treatment (T1). In all cases, a standard preadjusted edgewise technique was used in both dental arches after full mouth scaling. Three weeks after the placement of the appliance, second scoring was done (T2). After the second scoring, the patients were divided into two groups, each group consisting of 20 patients. Group I-herbal dentifrice and mouth wash and group II-nonherbal dentifrice and mouthwash. 3 weeks after the use of herbal and nonherbal dentifrices, the third scoring (T3) was done.

Results: Between T1 and T2, there was a significant increase in PI $(p=0.005)$ and significant decrease in GI $(p=0.000)$. At T3, no significant difference in $\mathrm{PI}$ or $\mathrm{Gl}$ was found $(p=0.051)$.

Conclusion: The present study has proved that there is no difference in the type of dentifrice, i.e., herbal or nonherbal for the effectiveness of oral hygiene. Both the types of dentifrice provided adequate oral hygiene maintenance.

Clinical significance: More importance to the method of brushing should be given than the type of dentifrices.

Keywords: Gingival index, Herbal dentrifrices, Nonherbal dentrifrices, Oral hygiene, Plaque index.

Journal of Oral Health and Community Dentistry (2019): 10.5005/jp-journals-10062-0059
\end{abstract}

\section{INTRODUCTION}

The root cause for most of the commonest oral health problems, such as dental caries, gingivitis, periodontitis, halitosis, etc. is dental plaque. Dental plaque also known as tooth plaque, microbial plaque, and dental biofilm, is a soft, sticky film that builds up on the teeth. Mechanical aids, such as tooth brushes, mouth rinses, and dentifrices ${ }^{1}$ are commonly used to remove dental plaque.

In fixed orthodontic patients, the presence of brackets and arch wires acts as a significant stagnation area for plaque deposits. Hence, conventional oral hygiene is difficult. This can result in demineralization, such as white spot lesions, dental caries and gingivitis, periodontitis, etc. ${ }^{2}$ Hence, assessment of oral hygiene for a fixed orthodontic patients is a must at every visit and for this most routinely, visual assessment of plaque with ordinal indexes, such as Silness and $\mathrm{Loe}^{3}$ is done.

Various dentifrices and mouth rinses are available in the market, which are used for maintaining oral hygiene. Chemicals such as triclosan and chlorhexidine are an important component in dentifrices and mouth rinses, which helps in preventing plaque and gingivitis. ${ }^{4}$ However, some of these chemicals cause alterations in taste sensation and cause tooth staining. ${ }^{5}$ This has given scope for research on the formulation of many natural, nonchemical, and herbal dentifrices. These natural ingredients are known to have anti-inflammatory, analgesic, and antiseptic effects. ${ }^{6}$

But there are very few studies researching the comparison of efficacy between herbal and nonherbal dentifrices and mouth washes in fixed orthodontic patients. Hence, this research study has been undertaken with the aim of evaluating the oral hygiene effectiveness between herbal and nonherbal dentifrice and mouth wash in fixed orthodontic cases.

\begin{abstract}
$\overline{\text { 1,4 Department of Orthodontics, JSS Dental College and Hospital, }}$ Mysuru, Karnataka, India

${ }^{2}$ Department of Periodontics, JSS Dental College and Hospital, Mysuru, Karnataka, India

${ }^{3}$ Department of Pediatric Dentistry, JSS Dental College and Hospital, Mysuru, Karnataka, India

Corresponding Author: Bhagyalakshmi Avinash, Department of Orthodontics, JSS Dental College and Hospital, Mysuru, Karnataka, India, Phone: +91 9902764937, e-mail:drbhagya_la@yahoo.co.in

How to cite this article: Avinash B, Shivamallu AB, Bhojraj N, et al. Comparison of Reduction of Dental Plaque and Gingivitis between Herbal and Nonherbal Dentifrices and Mouth Rinses in Orthodontic Cases: A Randomized Single-blind Clinical Study. J Oral Health Comm Dent 2019;13(3):69-72.

Source of support: The trail is financially supported by JSS Academy of Higher Education and Research, Mysore as minor research grant

Conflict of interest: None
\end{abstract}

\section{Materials and Methods}

This clinical trial was undertaken at JSS Dental College and Hospital, JSS Academy of Higher Education and Research, Mysuru.

\section{Source of the Data}

In this study, 40 patients from the out patients in the Department of Orthodontics and Dentofacial Orthopedics, JSS Dental College and Hospital, Mysuru requiring fixed orthodontic treatment was selected. 
The inclusion criteria are as follows:

- Age between 13 years and 21 years.

- Normal gingiva without inflammation, bleeding, and microbial plaque on teeth.

The exclusion criteria are as follows:

- Prior use of any antibiotic or mouthwash for 10 consecutive days in the last 3 months.

- A history of sensitivity to any mouthwash.

- The use of corticosteroids in the last 3 months.

- Severe gingival inflammation during the study.

- Poor cooperation reported by a parent of subjects.

- Systemic diseases and conditions affecting the gingival health.

- Expecting and nursing mothers.

The study was a single center, single blind, parallel arms, randomized controlled clinical trial with an allocation ratio of 1:1. The study was approved by the Institutional Ethics Committee, JSS Dental College and Hospital, Mysuru (Ref. No: JSS/DC/Ethical/11-12 dated, 11-02-2012). The study with possible discomforts, benefits, and harms were clearly explained to the participants and informed consent was sought. The study was conducted in the clinic of the Department of Orthodontics and Dentofacial Orthopaedics, JSS Dental College and Hospital, Mysuru with the above-mentioned inclusion and exclusion criteria.

The sample size was calculated based on the formula for comparison of means. The type I error $(\alpha)$ was set at $95 \%$ and type II error (power of the study) $\beta$ was set at $80 \%$. A variance $\sigma$ of 0.24 was derived by pooling the variance and obtaining the average of the gingival index (GI) scores from previous studies. The expected minimal difference was set at 0.2. A sample size of 20 per group was estimated for the study and the total sample size required was 40 .

All the participants were screened for inclusion and exclusion criteria. To randomly allocate the participants to either of the groups, fish bowl method was used where in the participants were asked to pick a chit placed in a bowl. The chits were numbered (1-40). These numbers were randomly assigned to either of the group by an investigator who was not involved in the clinical examination of the patient.

All the clinical examination was conducted by the principal investigator. In order to blind the principal investigator, the dentifrices and mouth washes were masked with an opaque cover. These dentifrices and the mouth wash were preassigned to match with chit number picked up by the participant. This way even the participants were unaware about the group to which they belonged.

For all the participants, oral hygiene status was assessed at three occasions during the study. The first assessment of PI and GI was done before starting the orthodontic treatment (T1). This included scoring the amount of dental plaque according to the plaque index system (P1I) of Silness and Loe (1964) on the day of examination, the patients were instructed to refrain from tooth brushing and use of mouthwash. Gingival status was assessed by scoring the gingival inflammation using the GI of Loe and Silness (1963).

In all cases, a standard preadjusted edgewise technique was used in both dental arches after full mouth scaling. 3 weeks after the placement of the appliance, second scoring was done (T2). After the second scoring, the patients were divided in to two groups, each group consisting of 20 patients:
Group I: herbal dentifrice and mouth wash.

Group II: nonherbal dentifrice and mouth wash.

Dentifrice, soft bristles tooth brush and mouth wash was distributed, and the subjects were instructed to brush their teeth with modified Bass technique for 3 minutes two times daily and use the mouthwash half an hour after brushing the teeth. They were advised to use them according to the instructions on the brochures after a thorough explanation. 3 weeks after the use of herbal and nonherbal dentifrices, the third scoring (T3) was performed.

\section{Statistical Methods}

All data were analyzed using SPSS version 20.0. Paired $t$ test was used for comparison between baseline (T1) and second follow-up (T2). Independent sample $t$ test was used for intergroup comparisons (at T3).

\section{Results}

A total of 40 participants were included in the study. After T2, they were randomly assigned to either group I or II with 20 participants each in the group (Flowchart 1). Participants who belonged to group I received herbal dentifrice and mouthwash and for those participants who belonged to group II receive nonherbal and mouthwash. Grouping of the participants was done randomly with blinding of the principal investigator.

Table 1 shows the descriptive statistics of the participants of the study. Between T1 and T2, there was a significant increase in $\mathrm{PI}(p=0.005)$ and significant decrease in $\mathrm{Gl}(p=0.000)$ (Table 2

Flowchart 1: Flow chart of the methodology

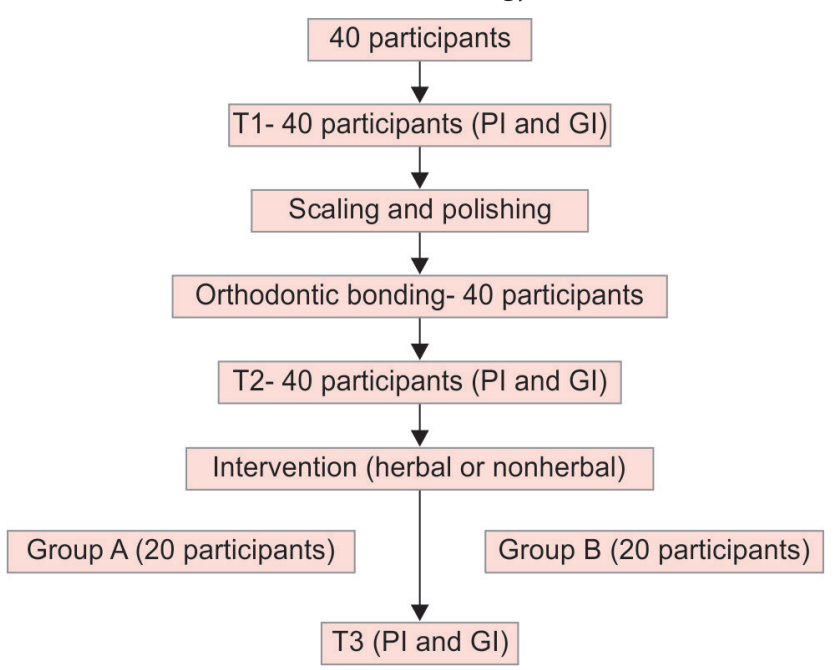

Table 1: Descriptive statistics of the study participants

\begin{tabular}{lllll}
\hline & & $n$ & Mean & SD \\
\hline GI & T1 & 40 & 1.5 & 0.03 \\
& T2 & 40 & 1.4 & 0.25 \\
PI & T1 & 40 & 2.0 & 0.05 \\
& T2 & 40 & 2.1 & 0.05 \\
GI & Group I & 20 & 1.4 & 0.03 \\
& Group II & 20 & 1.3 & 0.04 \\
PI & Group I & 20 & 1.9 & 0.67 \\
& Group II & 20 & 1.8 & 0.83 \\
\hline
\end{tabular}


Table 2: Statistically significant increase in plaque index and decrease in gingival index after orthodontic bonding

\begin{tabular}{lclrll}
\hline \multicolumn{2}{l}{ Paired sample test } \\
\hline & Mean & Std & \multicolumn{1}{l}{$T$} & $d f$ & Sig (two-tailed) \\
\hline GI (T1-T2) & 0.6425 & 0.13519 & 3.006 & 39 & 0.005 \\
PI (T1-T2) & -0.04775 & 0.03445 & -8.765 & 39 & 0.000 \\
\hline
\end{tabular}

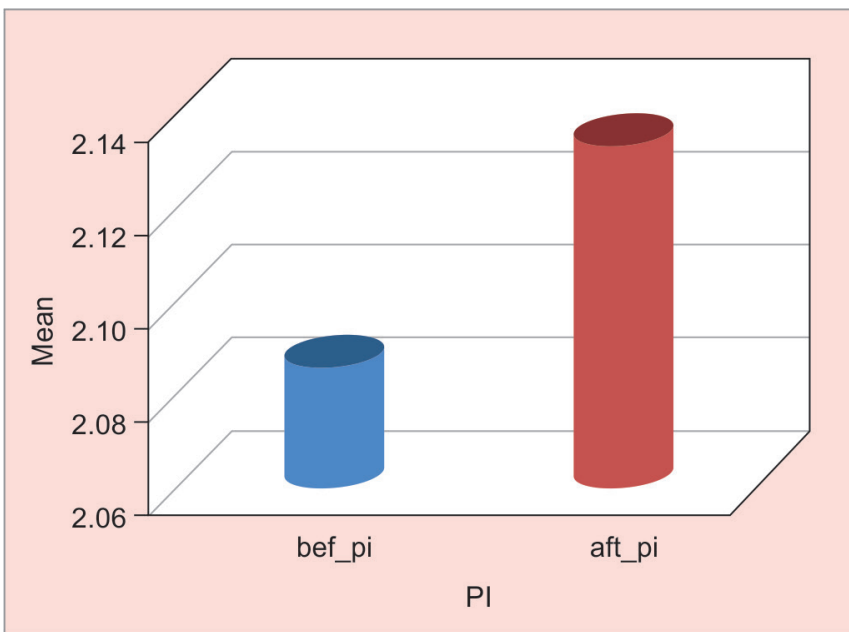

Fig. 1: Statistically significant increase in plaque index after orthodontic bonding

Table 3: No significant difference in plaque index and gingival index after grouping

\begin{tabular}{lllll}
\hline \multicolumn{5}{l}{ Independent sample test } \\
\cline { 2 - 4 } & \multicolumn{4}{c}{ t test for equality of means } \\
& $t$ & $d f$ & $\begin{array}{l}\text { Sig } \\
\text { (two-tailed) }\end{array}$ & Mean difference \\
\hline $\mathrm{Gl}$ & 1.001 & 38 & 0.323 & 0.05100 \\
$\mathrm{PI}$ & 0.475 & 38 & 0.638 & 0.05100 \\
\hline
\end{tabular}

and Fig. 1). At T3, no significant difference in $\mathrm{PI}$ or $\mathrm{Gl}$ was found $(p=0.051)$ (Table 3).

\section{Discussion}

Dental plaque deposits on teeth is a concern for both cosmetic and pathogenic nature. The presence of plaque may be the culprit for dental caries, gingivitis, periodontal problems, and halitosis. Many mechanical aids are used worldwide to remove or control plaque, including tooth brushes, dental floss, mouth rinses, and dentifrices. Mechanical plaque removal is one of the most accepted methods for controlling plaque and gingivitis. However, it is expected that less than one-third of the population can effectively perform mechanical plaque removal.

Our study evaluated the reduction of dental plaque and gingivitis between herbal and nonherbal dentifrice and mouthwash in orthodontic cases. Patients undergoing fixed orthodontic therapy may have difficulty maintaining good oral hygiene not only due to the increased plaque accumulation around the brackets and wires but also the difficulty posed by the appliances in accessing such areas. The retentive nature of appliances in patients undergoing fixed orthodontic therapy and difficulty in accessing certain areas severely affects the ability to maintain a good oral hygiene. Maintenance of oral hygiene in orthodontic patients can be conducted by both clinical and supplementary methods of care. A variety of products, such as mouth rinses, dentifrices, and so on have been used as agents to prevent plaque accumulation and gingivitis. Several chemical preventive agents have beneficial effects in the control of plaque and to reduce or prevent oral disease. Hence, various chemical formulations were tried in dentifrices. Chemicals mainly triclosan and chlorhexidine have been added in the mouth rinses and dentifrices to prevent plaque and gingivitis. However, some of these substances show undesirable adverse effects such as tooth staining and altered taste. This had led to paying increased attention on using natural ingredients in herbal dentifrices. Herbal ingredients have several benefits; chamomile has anti-inflammatory effects echinacea has immune stimulatory property, sage, and rhatany have antihemorrhagic properties, myrrh is a natural antiseptic; peppermint oil has analgesic, antiseptic, and anti-inflammatory properties. Hence, the aim of the study was to evaluate the reduction of dental plaque and gingivitis between herbal and nonherbal dentifrice, and mouthwash in orthodontic cases.

All 40 study participants completed the study period. Between $\mathrm{T} 1$ and $\mathrm{T} 2$, there was a significant increase in $\mathrm{PI}(p=0.005)$ and significant decrease in $\mathrm{Gl}(p=0.000)$. The increase in PI can be explained due to the presence of orthodontic brackets and wires. These orthodontic brackets and wires act as retention for plaque and makes it difficult for effective oral hygiene maintenance. This is in accordance with studies by Rustic, Davies, and Thornberg. ${ }^{7-13}$ The reduction in $\mathrm{Gl}$ can be due to the scaling and polishing effect that was performed on all the participants after their recruitment. This is in accordance with studies by Copulos, Hunter, and Nishimine. ${ }^{14-18}$

After the intervention was introduced among the participants in terms of providing herbal and nonherbal dentifrice, i.e., group and group II, no significant difference in $\mathrm{PI}$ or GI was found. This is in accordance with studies by Ozaki, Sushma, Saxer and Mullaly. ${ }^{19-22}$ This means that the type of dentifrice had no effect on the oral hygiene status of the participant. Hence, it must be emphasized that more than the type of dentifrice to be prescribed it is the method of brushing, which is important for the maintenance of effect oral hygiene. In this study, Bass method of brushing was advocated in the patients.

\section{Summary and Conclusion}

The present study has proved that there is no difference in the type of dentifrice, i.e., herbal or nonherbal for the effectiveness of oral hygiene. Both the types of dentifrice provided adequate oral hygiene maintenance. Hence, more than the type of dentifrice, much emphasis must be given to the method of brushing.

Colgate total toothpaste contains triclosan and fluoride. Triclosan has antibacterial properties; it is well effective against plaque and gingivitis. Fluoride has anticaries properties.

The herbal toothpaste contains Akarkara (Anacyclus pyrethrum), neem (Azadirachta indica), Babool (Acacia arabica), Pudina (Menthe spicata), Long bud (Syzygium aromaticam), Tomar (Xanthoxylum alatum), Haldi (Curcuma longa), Pilu (Salvadora persica), Bakul (Mimusops elengi), Vidang (Embelia ribes), etc. The above herbal ingredients have the known effect of antibacterial, antiinflammatory, and anticariogenic properties.

Based on the results obtained, it can be concluded that clinically herbal dentifrices are as effective as nonherbal (conventional) 
dentifrices in the reduction of plaque, gingivitis, and salivary neutrophil count. Therefore, both herbal and nonherbal products are equally effective and can may be advised to be used in patients undergoing orthodontic therapy.

\section{References}

1. Barnes VM, Richter R, DeVizio W. Comparision of the short-term antiplaque/antibacterial efficacy of two commercial dentifrices. J Clin Dent 2010;21(4):101-104.

2. Ogaard B, ten Bosch JJ. Regression of white spot enamel lesions. A new optical method for quantitative longitudinal evaluation in vivo. Am J Orthod Dentofac Orthop 1994;106(3):238-242. DOI: 10.1016/ S0889-5406(94)70042-7.

3. Silness $\mathrm{P}$, Loe H. Periodontal disease in pregnancy. Acta Odontol Scand 1962;22:121-135. DOI: 10.3109/00016356408993968.

4. George J, Hegde S, Rajesh KS, et al. The efficacy of a herbal-based toothpaste in the control of plaque and gingivitis: a clinic-biochemical study. Indian J Dent Res 2009;20(4):480-482. DOI: 10.4103/09709290.59460.

5. de Oliveira SM, Torres TC, Pereira SL, et al. Effect of dentifrice containing aloe vera on plaque and gingivitis control. a double-blind clinical study in humans. J Appl Oral Sci 2008;16(4):293-296. DOI: 10.1590/S1678-77572008000400012.

6. Radafshar G, Mahboob F, Kazemnejad E. A study to assess the plaque inhibitory action of herbal-based toothpaste: a double blind controlled clinical trial. J Med Plants Res 2010;4(12):1182-1186.

7. Ristic M, Vlahovic Svabic M, Sasic M, et al. Clinical and microbiological effects of fixed orthodontic appliances on periodontal tissues in adolescents. Orthod Craniofac Res 2007;10(4):187-195. DOI: 10.1111/j.1601-6343.2007.00396.x.

8. Davies TM, Shaw WC, Worthington HV, et al. The effect of orthodontic treatment on plaque and gingivitis. Am J Orthod Dentofacial Orthop 1991;99(2):155-161. DOI: 10.1016/0889-5406(91)70118-G.

9. Thornberg MJ, Riolo CS, Bayirli B, et al. Periodontal pathogen levels in adolescents before, during, and after fixed orthodontic appliance therapy. Am J Orthod Dentofacial Orthop 2009;135(1):95-98. DOI: 10.1016/j.ajodo.2007.02.057.

10. Bollen AM, Cunha-Cruz J, Bakko DW, et al. The effects of orthodontic therapy on periodontal health: a systematic review of controlled evidence. J Am Dent Assoc 2008;139(4):413-422. DOI: 10.14219/jada. archive.2008.0184.

11. Preoteasa $\mathrm{CT}$, lonescu E, Preoteasa E. Risks and complications Associated with orthodontic treatment. In: Basic Aspects and Clinical Considerations Bourzgui Farid 2012.

12. Talic NF. Adverse effects of orthodontic treatment: a clinical perspective. Saudi Dent J 2011;23(2):55-59. DOI: 10.1016/j. sdentj.2011.01.003.

13. Dannan A. An update on periodontic-orthodontic interrelationships. J Indian Soc Periodontol 2010;14(1):66-71. DOI: 10.4103/0972$124 X .65445$.

14. Copulos TA, Low SB, Walker CB, et al. Comparative analysis between a Modified ultrasonic tip and hand instruments on clinical parameters of periodontal disease. J Periodontol 1993;64(8):694-700. DOI: 10.1902/jop.1993.64.8.694.

15. Hunter RK, O'Leary TJ, Kafrawy AH. The effectiveness of hand versus ultrasonic instrumentation in open flap root planing. J Periodontol 1984;55(12):697-703. DOI: 10.1902/jop.1984.55.12.697.

16. Nishimine $D, O^{\prime}$ Leary TJ. Hand instrumentation versus ultrasonics in the removal of endotoxins from root surfaces. J Periodontol 1979;50(7):345-349. DOI: 10.1902/jop.1979.50.7.345.

17. Adriaens PA, Adriaens LM. Effects of nonsurgical periodontal therapy on hard and soft tissues. Periodontol 2000 2004;36:121-145. DOI: 10.1111/j.1600-0757.2004.03676.x.

18. Cobb CM. Clinical significance of non-surgical periodontal therapy: an evidence-based perspective of scaling and root planing. J Clin Periodontol 2002;29(Suppl. 2):6-16.

19. Ozaki F, Pannuti CM, Imbronito AV, et al. Efficacy of an herba tooth paste on patients with established gingivitis - a randamised controlled trial. Braz Oral Res 2006;20(2):172-177. DOI: 10.1590/S180683242006000200015.

20. Sushma S, Nandlal B, Srilatha KT. A comparative evaluation of a commercially available herbal and non-herbal dentifrice on dental plaque and gingivitis in children - a residential school based oral health programme. J Dent Oral Hyg 2011;3:109-113.

21. Saxer UP, Menghini G, Bohnert KJ, et al. The effect of two toothpastes on plaque and gingival inflammation. J Clin Dent 1995;6(2):154-156.

22. Mullally BH, James JA, Coulter WA, et al. The efficacy of a herbal-based toothpaste on the control of plaque and gingivitis. J Clin Periodonto 1995;22(9):686-689. DOI: 10.1111/j.1600-051X.1995.tb00827.x. 